\title{
Automating Hydraulic Engineering Experiments
}

\author{
Stuart CAMERON \\ University of Aberdeen, School of Engineering, Aberdeen, United Kingdom \\ s.cameron@abdn.ac.uk
}

\begin{abstract}
Automating hydraulic engineering experiments may allow an increased number of scenarios or repetitions to be studied within a research programme and additionally increase measurement precision by reducing human errors. Several aspects of the Aberdeen Open Channel Facility (AOCF) have already been automated, including flow configuration and control and stereoscopic particle image velocimetry deployment and calibration. We expect these developments to contribute to scientific progress through enabling larger systematic data sets in future studies.
\end{abstract}

Keywords: automation, particle image velocimetry, open-channel flume.

\section{INTRODUCTION}

Hydraulic engineering research often involves a large parameter space requiring systematic experiments to fully uncover underlying physical mechanisms. For example, uniform, steady, open-channel flow over a rough bed may be characterised by the flow depth $(H)$, the shear velocity $\left(u^{*}\right)$, the depth averaged velocity $(U)$, the channel width $(B)$, the bed slope $\left(S_{b}\right)$, the fluid density and viscosity ( $\rho$ and $v$ ), the roughness height or particle size $(\Delta)$, and potentially additional length scales describing the roughness geometry. To study hydraulic resistance or turbulence structure, for example, the number of experiments required to explore the relevant parameter space may be unachievable with traditional laboratory flumes and measurement technologies. These restrictions on the amount of data that can be captured during a research programme ultimately limit scientific progress. In this paper, we explore how automating aspects of hydraulic engineering experiments can address this shortfall, with particular focus on automating PIV deployment and flume setup and configuration. 


\section{AUTOMATING HYDRAULIC FLUMES AND INSTRUMENTATION}

Automation in this context means to bring aspects of an experiment under the control of a computer software programme. One reason to attempt this is to reduce experiment setup time and reduce down-time between experiments. Automation, however, has additional benefits in that experiment methodology is precisely defined by a computer programme. Therefore, results are highly repeatable, human error is minimised and produced data is of higher quality. At the University of Aberdeen we have been exploring how automation can support our research projects. In the following, a few examples of our progress to date are outlined.

\subsection{Particle image velocimetry}

Stereoscopic particle image velocimetry poses several challenges that must be overcome before it can be programmatically controlled to deploy across a sequence of measurement positions. Firstly, "water prisms" attached to flume sidewalls are conventionally used to limit internal reflection and image distortion, however, they prevent the measurement plane from being traversed. Secondly, a typical calibration procedure involves creating near still-water conditions in the channel, then taking pictures of a dot grid calibration plate manually positioned at several different points within the camera field of view. We have addressed these challenges by designing a "semi immersible" camera lens and implementing a single point calibration technique. The semi-immersible lens was designed such that a prism at the tip of the lens just penetrates the water surface. The camera can then be traversed within the channel, free from the constraints of the water prisms. The calibration plate in our setup was replaced with a height-adjustable back-lit pinhole that can be deployed through the flume bed, even when the flow is running. We then traverse the cameras, taking pictures of the pinhole to generate the data required to compute a pinhole camera calibration model. This calibration approach can be run automatically after an experiment without manual intervention. We have used this setup in the AOCF to measure double averaged velocity field statistics over rough beds.

\subsection{Open-channel flume}

Establishing a uniform flow in a laboratory open-channel flume with a prescribed flow depth $(H)$ and shear velocity $(u *)$ involves calculating and setting the required bed slope $S_{b}=u *^{2} /[g H]$, where $g$ is acceleration due to gravity, and then experimentally adjusting the flowrate and weir setting until uniform flow at the correct water level is found. Automating this procedure involves firstly bringing the pumps, flume slope, and weir mechanisms under computer control. This can be achieved using relatively simple hardware and software systems. To measure flow depth profiles along the channel and check flow uniformity, we measure both bed and water surface profiles with an array of confocal chromatic sensors attached to a traverse mechanism. This instrument has a footprint of around 20 microns, sub-micron resolution, and very little drift making it ideal to capture precise flow depth measurements. The final step to automate flow setup is the software control loops to adjust pump speed and weir setting based on the feedback from the confocal sensors. We hope to bring this last step online within 2021. 